\title{
Australian Diabetes Foot Network: management of diabetes-related foot ulceration - a clinical update
}

Shan M Bergin BAppSci(Pod), PhD, Podiatrist

Joel M Gurr BSc(Pod), MBA Podiatrist

Bernard P Allard MB BS, FRACS(Vasc) Vascular Surgeon

Emma L Holland RN, CDE, MA(Ed), Diabetes Educator

Mark W Horsley MB BS, FRACS(Ortho) Orthopaedic Surgeon

Maarten C Kamp FRACP, MHA, GAICD, Endocrinologis

Peter A Lazzarin BAppSci(Pod) Podiatrist

Vanessa L Nube DipAppSci(Pod), MSc(Med) Podiatrist

Ashim K Sinha MB BS, MD, FRACP. Endocrinologist

Jason T Warnock DipAppSc(Chir) GradCertDiabEd Podiatrist

Jan B Alford RN, MEd(AdEd), CDE Diabetes Educator

Paul R Wraight MB BS, FRACP, PhD, Endocrinologist

Australian Diabetes Foot Network, Australian Diabetes Society,

Sydney, NSW.

paul.wraight@ mh.org.au

MJA 2012; 197: 226-229 doi: 10.5694/miall.10347 n 2005, over 1000 people with diabetes died as a direct result of lower limb ulceration; this represented $8 \%$ of all diabetes-related deaths. ${ }^{1}$ Annually, there are about 10000 hospital admissions for diabetes-related foot ulcers (DRFUs) in Australia, with lower limb amputation a common outcome. ${ }^{1,2}$

Most DRFUs occur in the presence of peripheral sensory neuropathy, foot deformity and/or trauma, with peripheral arterial disease (PAD) and infection being further complicating factors that prevent or delay ulcer healing. ${ }^{3-5}$ Therefore, for successful management of DRFUs, all interdependent factors affecting healing should be addressed simultaneously. ${ }^{6}$ Health professionals without the resources, time and/or clinical skills to appropriately manage these individuals must refer them to others that do have the resources and skills. People with complex or non-healing DRFUs should be referred to a multidisciplinary foot care team (MFCT) comprising medical, surgical, nursing, podiatry and other allied health professionals with appropriate skills and knowledge, for immediate management.

\section{Assessment}

All individuals with a DRFU should be clinically assessed for aetiology and factors that may prevent wound healing. A comprehensive foot and wound assessment (Box 1), complemented by a more general assessment, will help guide investigation and management strategies. ${ }^{6,7} \mathrm{Pub}-$ lished wound classification systems are a useful adjunct to clinical assessment and can assist with wound documentation and assessing progress over time. The Wagner and University of Texas classification systems are two readily available examples. ${ }^{8,9}$

The initial response to treatment can be a robust predictor of wound healing. ${ }^{10}$ In the absence of clear benchmarks for DRFU healing times, we suggest that wounds demonstrating a lack of progress in healing after 4 weeks of appropriate treatment should be referred to an MFCT. Indicators for more immediate referral to an MFCT or emergency department include gangrene, limb-threatening ischaemia, deep ulcers (bone, joint or tendon in the wound base), ascending cellulitis, systemic symptoms of infection and abscesses.

\section{Management recommendations}

General management recommendations are summarised in Box 2.

\section{Debridement}

Debridement of DRFUs is an essential component of wound bed preparation, as it removes non-viable tissue, allows

\section{Summar \\ Appropriate assessment and management of diabetes- related foot ulcers (DRFUs) is essential to reduce amputation risk. \\ Management requires debridement, wound dressing, pressure off-loading, good glycaemic control and potentially antibiotic therapy and vascular intervention. \\ - As a minimum, all DRFUs should be managed by a doctor and a podiatrist and/or wound care nurse. \\ Health professionals unable to provide appropriate care for people with DRFUs should promptly refer individuals to professionals with the requisite knowledge and skills. \\ - Indicators for immediate referral to an emergency department or multidisciplinary foot care team (MFCT) include gangrene, limb-threatening ischaemia, deep ulcers (bone, joint or tendon in the wound base), ascending cellulitis, systemic symptoms of infection and abscesses. \\ Referral to an MFCT should occur if there is lack of wound progress after 4 weeks of appropriate treatment.}

comprehensive examination of the wound bed and assessment of actual wound size, has the potential to convert a chronic wound to an acute wound, and reduces local pressure on the ulcer. ${ }^{10,11}$ Surgical sharp debridement is required for management of deep necrotic tissue, gangrene, drainage of collections, deep infections or when pain necessitates the use of an anaesthetic. ${ }^{12,13}$ Non-surgical sharp debridement is generally required every 1-2 weeks to remove non-viable tissue, including the surrounding callus. ${ }^{12}$ Non-sharp debridement (enzymatic, autolytic and biological) can be used as an adjunct to sharp debridement and can be especially helpful for adherent slough, or when pain limits non-surgical sharp debridement. ${ }^{14}$

In the presence of PAD, debridement could potentially create a larger non-healing wound, therefore vascular investigations are indicated before debridement. One exception is for an abscess, which requires immediate drainage to reduce tissue pressures, control infection and prevent further tissue loss. Same-day vascular review is then recommended.

\section{Dressing selection}

Ulcers should be regularly inspected and cleaned with saline or clean water. ${ }^{7}$ Cleaning with surface antiseptics (eg, chlorhexadine) is not recommended, as this may be harmful to granulation tissue. ${ }^{11}$

There is no evidence showing that one type of dressing is better than another, or that one type of dressing can be used on all wounds. Dressing choice will generally change as the wound characteristics change during healing. Dressing selection is based on the principles of "moist wound healing", where inflammation, infection and exudate are 


\section{Wound and foot assessment}

Both feet should be assessed for:

- abnormal foot shape, previous amputation and new ulceration

- signs of infection

peripheral sensory neuropathy

peripheral arterial disease

possible cause of ulceration (footwear)

All ulcers should be assessed for the following at each contact:

site, size and depth

probable bone in the wound base

presence and amount of slough, necrotic and granulation tissue

presence of exudate (type and amount)

presence or absence of pain

signs of clinical infection

condition of tissue and skin surrounding ulcer head resection and tenotomies may improve healing time and reduce the risk of ulcer recurrence. ${ }^{19}$ Surgery of this type should not be considered in the presence of PAD without input from a vascular specialist.

Ongoing relief of pressure is important for secondary prevention in people with healed foot ulceration, particularly in the presence of a foot deformity. In the absence of a foot deformity, most individuals with a healed ulcer can be accommodated in off-the-shelf footwear (unmodified comfort or sports shoes). Medical-grade footwear is generally required for people with a foot deformity as it provides extra depth, multiple-width fittings and features designed for a broader range of foot types. Medical-grade footwear may also be fitted with custom-made orthoses to redistribute plantar pressure and reduce the risk of future foot ulceration. Medical-grade footwear should be purchased from suppliers with trained staff. Medical-grade footwear can be expensive, so health professionals should be aware of government-funded schemes to support such purchases (eg, the Department of Veterans Affairs "gold cards").

\section{Infection}

\section{Soft tissue infection}

Soft tissue infection is a common complication of DRFUs and must be treated immediately with systemic antibiotics. Clinical signs and laboratory markers typically associated with infection can be blunted or absent in people with diabetes, so should not be relied on in isolation to assess infection severity. ${ }^{20}$ An increase in skin pigmentation may be a sign of inflammation and/or infection in individuals with pigmented skin.

Clinically infected wounds should be cultured by deep tissue swabs taken after debridement or by tissue samples, for identification of microorganisms and antibiotic sensitivities. ${ }^{21}$ Mild, acute wound infections (characterised by $<2 \mathrm{~cm}$ of surrounding cellulitis) can usually be managed by primary health care professionals, using oral antibiotics effective for gram-positive cocci (eg, Staphylococcus aureus and streptococci). Infections characterised by systemic toxicity, more than $2 \mathrm{~cm}$ of cellulitis, a deep abscess or osteomyelitis require immediate referral to hospital or an MFCT.

Wound chronicity, prior antibiotic use, renal impairment and hospitalisation are all risk factors for polymicrobial infections with gram-positive cocci, gram-negative bacilli and/or anaerobes. When these risk factors are present, broad-spectrum antibiotic therapy should immediately be commenced and then modified according to culture results. ${ }^{22}$ Clinically non-infected wounds require neither wound culture nor antimicrobial therapy. ${ }^{21}$

\section{Management of diabetes-related foot ulcers}

All diabetes-related foot ulcers will require a management "package" comprising:

- debridement of non-viable tissue

- wound dressings

pressure off-loading

antibiotics when clinical infection is present

management of peripheral arterial disease when present

management of glycaemic control and other cardiovascular comorbidities would benefit from surgical intervention. Procedures such as tendoachilles lengthening, arthroplasties, metatarsal 
3 Superficial ulcer on third toe resulting from hammer toe deformity and ill fitting footwear (scale in $\mathrm{cm}$ )

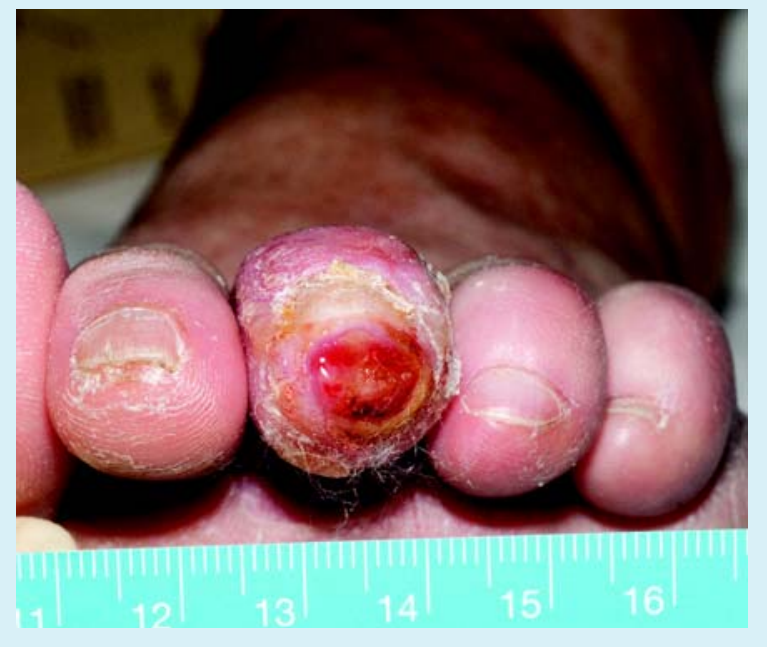

\section{Osteomyelitis}

Osteomyelitis can complicate DRFUs, increasing the risk of amputation. A positive "probe to bone" test is highly suggestive of osteomyelitis and should be performed routinely by inserting a sterile instrument into the ulcer to determine whether bone can be probed at the base. ${ }^{21,23}$ Plain $x$-ray is a valuable baseline investigation when osteomyelitis is suspected, and will also identify foreign bodies and tissue gas. The radiological signs of osteomyelitis may be delayed, so a normal x-ray should not be interpreted as definitive when there is a high level of clinical suspicion for osteomyelitis. Magnetic resonance imaging has a higher sensitivity and specificity for osteomyelitis than plain x-ray, so is the imaging modality of choice, and may help differentiate the condition from Charcot neuroarthropathy. ${ }^{21}$ Bone scans and white cell scans generally lack specificity for diagnosing osteomyelitis.

Although surgery has been the mainstay of treatment for pedal osteomyelitis, there is evidence supporting medical management of focal osteomyelitis with antibiotics or antibiotics combined with limited surgical resection. ${ }^{24-26}$ It is difficult to predict which cases will or will not respond to antibiotic therapy, so this strategy should be undertaken with the guidance and supervision of an MFCT.

It is worth noting that acute Charcot neuroarthropathy can mimic soft tissue infection and must be considered as a differential diagnosis in anyone with diabetic neuropathy who presents with a red, hot and/or swollen foot, especially in the absence of an ulcer.

\section{Vascular management}

If pedal pulses are absent in either foot, if there is gangrene or if there is no improvement in the ulcer healing despite appropriate wound management for 4 weeks, the person should be referred to a vascular specialist or an MFCT. Vascular specialists and MFCT members are the most appropriate individuals for selecting advanced investigations (eg, arterial Doppler ultrasound, computed tomography angiogram, magnetic resonance angiogram and catheter angiogram). Revascularisation by either percutaneous angioplasty or operative bypass is recommended whenever possible. ${ }^{27}$

\section{Glycaemic control and other cardiovascular comorbidities}

As well as specific treatment for a DRFU, it is important to undertake a general assessment of the individual's diabetes and other cardiovascular comorbidities. ${ }^{28}$ This is aimed at preventing the development or progression of other diabetes-related complications (which may further affect foot health), and at reducing mortality through modification of cardiovascular risk factors. Lipid and blood pressure control should be maximised, cessation of smoking encouraged and aspirin therapy considered. ${ }^{29}$ There is no study addressing the role of glycaemic control in the acute management of diabetes-related foot complications, but it is prudent to optimise glycaemic control at the time of acute illness, when there is infection and perioperatively. Metformin should be withdrawn when there is significant infection and/or ischaemia, when an individual is fasting for procedures and with administration of angiographic contrast. $^{29}$

\section{Multidisciplinary care}

It is well documented that best-practice management of complex DRFUs need coordinated, expert interdisciplinary input in both inpatient and outpatient settings. ${ }^{30,31}$ Management by MFCTs, comprising medical, surgical, nursing, podiatry and other allied health professionals with appropriate skills and knowledge, increases the percentage of healed ulcers and reduces wound healing times, hospitalisation and amputations. ${ }^{32,33}$

\section{Conclusion}

To achieve the best clinical outcomes, all DRFUs require multifactorial management from at least a medical practi-

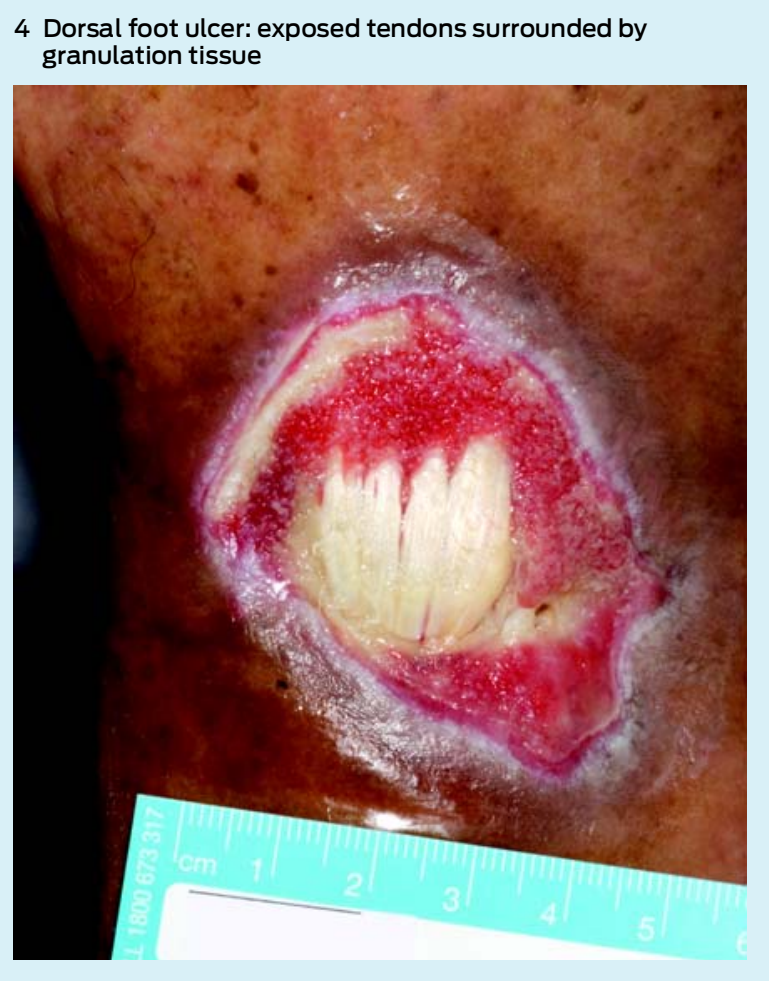


tioner with a podiatrist and/or wound care nurse. The management package requires appropriate wound debridement, wound dressings, pressure off-loading, glycaemic control and, when indicated, treatment of infection and improvement of peripheral blood flow. When there is significant PAD, infection or failure to respond to appropriate treatment, all individuals should promptly be referred to an MFCT or hospital.

Acknowledgements: The Australian Diabetes Society (ADS) receives funding from the National Diabetes Services Scheme. This guide is supported by the ADS, Australasian Podiatry Council, Australian Diabetes Educators Association, Australian and New Zealand Society for Vascular Surgery, Australian Wound Management Association and Diabetes Australia.

Competing interests: No relevant disclosures.

Provenance: Not commissioned; externally peer reviewed.

1 Australian Institute of Health and Welfare. Diabetes: Australian facts 2008. Canberra: AlHW, 2008. (AlHW Cat. No. CVD 40; Diabetes Series No. 8.) http:// www.aihw.gov.au/publication-detail/?id=6442468075 (accessed Apr 2011).

2 Davis WA, Norman PE, Bruce DG, Davis TM. Predictors, consequences and costs of diabetes-related lower extremity amputation complicating type 2 diabetes: the Fremantle Diabetes Study. Diabetologia 2006; 49: 2634-2641.

3 Reiber GE, Vileikyte L, Boyko EJ, et al. Causal pathways for incident lowerextremity ulcers in patients with diabetes from two settings. Diabetes Care 1999; 22: 157-162.

4 Currie CJ, Morgan C, Peters JR. The epidemiology and cost of inpatient care for peripheral vascular disease, infection, neuropathy, and ulceration in diabetes. Diabetes Care 1998; 21: 42-48.

5 Calhoun JH, Overgaard KA, Stevens CM, et al. Diabetic foot ulcers and infections: current concepts. Adv Skin Wound Care 2002; 15: 31-42.

6 American Diabetes Association. Consensus Development Conference on Diabetic Foot Wound Care. J Am Podiatr Med Assoc 1999; 89: 475-483.

7 Australian Wound Management Association Inc. Standards for wound management. 2nd ed. AWMA, 2010. http://awma.com.au/publications/2011 standards_for_wound_management_v2.pdf (accessed Apr 2012).

8 Armstrong DG, Lavery LA, Harkless LB. Validation of a diabetic wound classification system. The contribution of depth, infection, and ischemia to the risk of amputation. Diabetes Care 1998; 21: 855-859.

9 Wagner FW Jr. The dysvascular foot: a system for diagnosis and treatment. Foot Ankle 1981; 2: 64-122.

10 Sheehan $P$, Jones $P$, Caselli A, et al. Percent change in wound area of diabetic foot ulcers over a 4-week period is a robust predictor of complex healing in a 12-week prospective trial. Diabetes Care 2003; 26: 1879-1882.

11 European Wound Management Association. Wound bed preparation in practice [position document]. London: MEP Ltd, 2004. http://ewma.org/ fileadmin/user_upload/EWMA/pdf/Position_Documents/2004/pos_doc_ English_final_04.pdf (accessed Mar 2012).

12 Fisher TK, Scimeca CL, Bharara M, et al. A stepwise approach for surgical management of diabetic foot infections. J Am Podiatr Med Assoc 2010; 100: 401-405.

13 Steed DL, Donohoe D, Webster MW, Lindsley L; Diabetic Ulcer Study Group. Effect of extensive debridement and treatment on the healing of diabetic foot ulcers. J Am Coll Surg 1996; 183: 61-64.

14 Edwards J, Stapley S. Debridement of diabetic foot ulcers. Cochrane Database Syst Rev 2010; (1): CD003556. doi: 10.1002/14651858.CD003556.pub2.
15 Armstrong DG, Lavery LA; Diabetic Food Study Consortium. Negative pressure wound therapy after partial diabetic foot amputation: a multicentre, randomised controlled trial. Lancet 2005; 366: 1704-1710.

16 Bus SA, Valk GD, van Deursen RW, et al. The effectiveness of footwear and offloading interventions to prevent and heal foot ulcers and reduce plantar pressure in diabetes: a systematic review. Diabetes Metab Res Rev 2008; 24 Suppl 1: S162-S180.

17 Cavanagh PR, Bus SA. Off-loading the diabetic foot for ulcer prevention and healing. J Am Podiatr Med Assoc 2010; 100: 360-368.

18 Armstrong DG, Lavery LA, Wu S, Boulton AJM. Evaluation of removable and irremovable cast walkers in the healing of diabetic foot wounds: a randomised controlled trial. Diabetes Care 2005; 28: 551-554.

19 Frykberg RG, Bevilacqua NJ, Habershaw G. Surgical off-loading of the diabetic foot. J Am Podiatr Med Assoc 2010; 100: 369-384.

20 Lipsky BA. A report from the international consensus on diagnosing and treating the infected diabetic foot. Diabetes Metab Res Rev 2004; 20 Suppl 1: S68-S77.

21 Lipsky BA, Berendt AR, Deery HG, et al; Infectious Diseases Society of America. Diagnosis and treatment of diabetic foot infections. Clin Infect Dis 2004; 39: 885-910.

22 Antibiotic Expert Group. Therapeutic guidelines: antibiotic. Version 14 Melbourne: Therapeutic Guidelines Limited, 2010.

23 Lavery LA, Armstrong DG, Peters EJ, Lipsky BA. Probe-to-bone test for diagnosing diabetic foot osteomyelitis: reliable or relic? Diabetes Care 2007; 30: 270-274.

24 Berendt AR, Peters EJ, Bakker K, et al. Diabetic foot osteomyelitis: a progress report on diagnosis and a systematic review of treatment. Diabetes Metab Res Rev 2008; 24 Suppl 1: S145-S161.

25 Ha Van G, Siney H, Danan JP, et al. Treatment of osteomyelitis in the diabetic foot. Contribution of conservative surgery. Diabetes Care 1996; 19: 1257-1260.

26 Yadlapalli NG, Vaishnav A, Sheehan P. Conservative management of diabetic foot ulcers complicated by osteomyelitis. Wounds 2002; 14: 31-35. http:// www.woundsresearch.com/article/146?page=0,0 (accessed Mar 2012).

27 Ihnat DM, Mills JL Sr. Current assessment of endovascular therapy for infrainguinal arterial occlusive disease in patients with diabetes. J Am Podiatr Med Assoc 2010; 100: 424-428

28 Colagiuri S, Dickinson S, Girgis S, Colagiuri R. National evidence based guideline for blood glucose control in type 2 diabetes. Canberra: Diabetes Australia and NHMRC, 2009. http://diabetesaustralia.com.au/PageFiles/763/

Final\%20Blood\%20Glucose\%20Control\%20Guideline\%20August $\% 20200$ 9\%20\%282\%29.pdf (accessed May 2012).

29 American Diabetes Association. Standards of medical care in diabetes - 2010 Diabetes Care 2010; 33 Suppl 1: S11-S61.

30 Sumpio BE, Armstrong DG, Lavery LA, Andros G; SVS/APMA writing group. The role of interdisciplinary team approach in the management of the diabetic foot: a joint statement from the Society for Vascular Surgery and the American Podiatric Medical Association. J Vasc Surg 2010; 51: 1504-1506.

31 Apelqvist J, Bakker K, van Houtum WH, Schaper NC; International Working Group on the Diabetic Foot (IWGDF) Editorial Board. Practical guidelines on the management and prevention of the diabetic foot: based upon the International Consensus on the Diabetic Foot (2007) prepared by the International Working Group on the Diabetic Foot. Diabetes Metab Res Rev 2008; 24 Suppl 1: S181-S187.

32 Edmonds ME, Blundell MP, Morris ME, et al. Improved survival of the diabetic foot: the role of a specialised foot clinic. Quart J Med 1986; 60: 763-771.

33 Larsson J, Apelqvist J, Agardh C, Stenström A. Decreasing incidence of major amputation in diabetic patients: a consequence of a multidisciplinary foot care team approach? Diabet Med 1995; 12: 770-776.

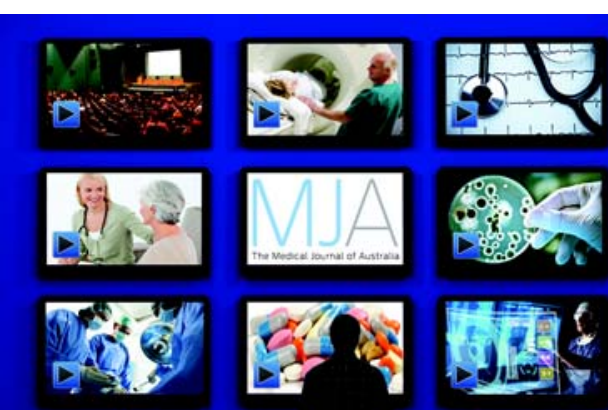

\section{MJA multimedia presentations}

The MJA invites authors to submit video and audio presentations relevant to the practice of clinical medicine in Australia for consideration for publication on the MJA website.

Multimedia presentations will be peer reviewed. Those considered to be of sufficient quality, academic rigour and relevance will be posted on the MJA website, with a brief summary being published in the MJA (citable in indexing services such as PubMed) linking to the presentation.

Video or audio presentations can be made specifically for the MJA

or can be lectures, seminars or conference presentations

(eg, clinical updates, demonstrations of clinical signs, or techniques). 